\title{
Resultados de las angioplastias del sector femoropoplíteo en isquemia crítica*
}

\section{Femoropopliteal angioplasty for the treatment of critical limb ischemia}

\author{
Drs. SANDRA HASBUN A. ${ }^{1}$, ROBERTO TAPIA R. ${ }^{1}$, PATRICIO TEPPER L. ${ }^{1}$, RAMÓN CELEDÓN L. ${ }^{1}$ \\ ${ }^{1}$ Equipo Cirugía Vascular Servicio de Cirugía Hospital Regional Talca, Chile
}

\begin{abstract}
RESUMEN
Introducción: La isquemia critica de las extremidades inferiores (EEII) es una condición que afecta a pacientes que presentan grave patología ateromatosa en múltiples territorios y por ende tienen mal pronostico vital. La terapia endovascular validada en algunos sectores, en éste no ha logrado posicionarse. Objetivo: Evaluar la evolución de los pacientes sometidos a Angioplastia Endoluminal Percutánea (AGP) del territorio femoropoplíteo por isquemia critica. Material y Método: Estudio retrospectivo que incluye 58 extremidades en 54 pacientes con isquemia critica de las EE.II por patología femoropoplítea, sometidos a angioplastia entre Julio del 2001 a Diciembre del 2004 y seguidos en su evolución hasta Abril del 2006. Resultados: Fueron realizadas 58 AGP (54 pacientes): masculinos 54\%, femeninos $46 \%$, y $74 \%$ diabéticos. Edad promedio 70,6 años. El grupo incluye lesiones TASC A, B, C y D. El $24 \%$ de las extremidades no tenía run off. Éxito técnico inicial se obtuvo en el $84 \%$. La permeabilidad primaria $43,3 \%$ a 1 año (ES 6,52), 32,7\% a 2 años (ES 7,44) y 16,9\% a 3 años (ES 6,31). Durante el periodo, 4 pacientes fueron sometidos a nuevas angioplastias del mismo territorio y 7 pacientes a by pass. Mortalidad a 30 días $1,7 \%$. Existieron 4 complicaciones mayores (8\%). Al término del seguimiento hay 20 amputaciones mayores (34\%), cuatro de ellas por compromiso local, las restantes por isquemia irreversible. La mortalidad al final del seguimiento fue de 29,6\%. Conclusión: La angioplastia infrainguinal en la isquemia crítica es posible, segura y de baja morbimortalidad, no excluyendo revascularizar mediante by pass si fracasa.
\end{abstract}

\section{PALABRAS CLAVE: Isquemia Critica, territorio femoropoplíteo, angioplastia.}

\section{SUMMARY}

Introduction: The critical ischemia of the lower extremities is a condition that affects patients who present serious atheromatous pathology in multiple territories and, consequently, have bad live prognosis. Endovascular therapy, valid in some sectors, has not been able to find a position in this one yet. Objectives: to evaluate the gradual development of patients who were subjected to angioplasty of the femoropopliteal territory because of critical ischemia. Material and method: retrospective study, which includes 58 extremities in 54 patients with critical ischemia of the interior extremities due to femoropopliteal pathology who were subjected to angioplasty between July 2001 and December 2004, and whose development was studied until

*Recibido el 5 de Junio de 2007 y aceptado para publicación el 12 de Octubre de 2007.

Correspondencia: Dra. Sandra Hasbun A.

1 Norte 13 Oriente $s / n$. Talca, Chile

e mail: sandra_hasbun@yahoo.com 
April 2006. Results: 58 AGP were performed in 54 patients: $54 \%$ male, $46 \%$ female, and $74 \%$ diabetics. The average age was 70.6 years old. The group includes TASC A, B, C, and D lesions. $24 \%$ of the extremities did not have run off. The initial technical success was obtained in $84 \%$ of the cases. The primary permeability $43,3 \%$ after a year (ES 6,52), 32,7\% after 2 years (ES 7,44) and 16,9\% after 3 years (ES 6,31). 4 patients were subjected to further angioplasty of the same territory and 7 patients were subjected to bypass during this period. The 30 -day mortality was $1,7 \%$. There were 4 major complications (8\%). By the end of the monitoring, there are 20 major amputations (34\%), 4 of these due to local compromise, and the rest of them due to irreversible ischemia. The mortality at the end of the monitoring was $29,6 \%$. Conclusion: infrainguinal angioplasty on critical ischemia is possible, safe and presents a low morbimortality, not excluding revascularization through bypass in case of failure.

\section{KEY WORDS: Critical ischemia, femoropopliteal, angioplasty.}

\section{INTRODUCCIÓN}

La isquemia crítica de las extremidades inferiores secundaria a patología arterial periférica crónica representa un importante porcentaje del quehacer quirúrgico de nuestro equipo.

El tratamiento endovascular de esta patología esperando el salvataje de la extremidad ha experimentado un rápido y explosivo crecimiento, con desarrollo de nuevas tecnologías que pretenden ofrecer soluciones efectivas y mínimamente invasivas; una intervención menor que puede incluso realizarse bajo anestesia local y que no impide la revascularización quirúrgica si esta fracasa.

Lo anterior resulta especialmente atractivo pues se trata de pacientes que tienen alta morbimortalidad asociada a procedimientos de revascularización tradicional y un mal pronostico vital ${ }^{1-3}$ con una mortalidad que puede llegar al $54 \%$ durante el primer año de seguimiento en los pacientes no revascularizados ${ }^{4}$.

La disparidad entre la terapia endovascular y los procedimientos abiertos, en relación a riesgos peri operatorios, confort del paciente, tiempo de recuperación y estadía hospitalaria están claramente a favor de la primera, pero la permeabilidad de la revascularización quirúrgica permanece superior en todos los territorios.

La Angioplastia Endoluminal Percutánea ha tenido una aceptación progresiva y ofrece distintos resultados en permeabilidad dependiendo del sector específico sobre el cual se realice, y de las características angiográficas de las lesiones, estando absolutamente validado en el manejo del sector aortoilíaco y existiendo aún controversia en territorios más dístales.

El objetivo de este trabajo es mostrar nuestra experiencia en AGP del sector femoropoplíteo en pacientes con isquemia crítica, evaluando; éxito técnico, mejoría clínica, permeabilidad, morbimortalidad y salvataje de la extremidad.

\section{MATERIAL Y METODO}

Fueron revisadas retrospectivamente, todas las fichas clínicas de los pacientes sometidos a AGP endoluminal por isquemia critica de las EEll con patología infrainguinal entre Julio del 2001 a Diciembre del 2004, siendo excluidos aquellos enfermos en quienes se realizó además algún procedimiento sobre el territorio Aorto llíaco.

Todos los pacientes fueron estudiados angiográficamente.

Se efectuó terapia endovascular a las extremidades con lesiones TASC A y B en forma preferente, y en las lesiones $C$ y $D$ que fueron incluidas se trató de pacientes con muy alto riesgo quirúrgico o sin material autólogo adecuado para cirugía.

Se analizaron las características demográficas del grupo, presentación clínica, resultados y complicaciones.

El seguimiento se llevó a cabo en todos los pacientes hasta la muerte o a Abril del año 2006.

Todas las AGP fueron realizadas en el pabellón de operaciones, empleando anestesia espinal o anestesia local en enfermos con muy alto riesgo. El acceso fue femoral ipsilateral en todos los casos, y se uso heparinización sistémica con $5000 \mathrm{U}$ EV luego de instalado el introductor hemostático.

Cuando más de una lesión fue tratada, la secuencia fue de proximal a distal o a la inversa según el operador.

Los pacientes continuaron con anticoagulantes orales luego del procedimiento solo si existía una razón médica previa para esto, de no ser así se indicó Aspirina $250 \mathrm{mg}$. diarios a permanencia.

Las lesiones fueron tratadas con balón de AGP convencional y sin stent.

Análisis de los datos: La categorización clínica de la isquemia se hizo de acuerdo a Recommended standards for reports dealing with lower extremity ischemia: revised version. ${ }^{5}$

Las lesiones fueron clasificadas según hallaz- 
gos angiográficos de acuerdo a TransAtlantic Inter Society Consensus (TASC) ${ }^{6}$. Calificando como mala salida distal si no existían vasos que entren al pie, regular solo un vaso y bueno 2 o 3 vasos. Se consideró fracaso técnico inicial cuando no se logra realizar la AGP por imposibilidad de cruzar la lesión con la guía o imposibilidad de reentrar al lumen luego de obtener un plano sub intimal.

El éxito de la AGP fue definido desde punto de vista técnico, anatómico, hemodinámico y clínico.

Esta fue anatómicamente exitosa en ausencia de estenosis residual igual o mayor al 30\%.

El éxito clínico fue referido con mejoría de los síntomas en al menos una categoría, y aumento del índice tobillo brazo en al menos 0,1 o aumento de la amplitud de la curva en $5 \mathrm{~mm}$ al Registro de Volumen de Pulso (PVR) en caso de presiones previas sobre lo normal.

La permeabilidad fue determinada en examen clínico por palpación de pulsos, y evaluados con índice tobillo brazo, PVR y su cálculo realizado según RVSRS 5 .

La recurrencia de los síntomas, deterioro en el estudio no invasivo o desaparición de pulsos distal a la lesión tratada fue asumido como pérdida de permeabilidad.

Las complicaciones fueron clasificadas según criterio $\mathrm{SVSRS}^{5}$, complicación mayor es la que causa muerte, deterioro permanente, requiere reoperación u otro procedimiento invasivo o prolonga la estadía hospitalaria. Amputación mayor se refiere a aquellas realizadas sobre el metatarso ${ }^{5}$.

Mortalidad operatoria fue definida como la ocurrida antes del alta o dentro de 30 días de efectuada la operación

\section{RESULTADOS}

Fueron realizadas 58 AGP en 54 pacientes. Masculinos 29 (54\%), Femenino 25 (46\%), durante un periodo de 41 meses, de Julio 2001 a Diciembre 2004, y seguida su evolución hasta Abril del 2006. (0 a 57 meses, con una media de 29.1 meses).

La media de edad fue de 70,6 DS 12,2 (rango de 37 a 94 años).

Los datos demográficos y características clínicas de los pacientes se resumen en la Tabla 1.

La indicación clínica fue en 23 extremidades $(40 \%)$ dolor de reposo (categoría 4), y en 35 extremidades $(60 \%)$ con ulcera o necrosis (categoría 5).

Del total 14 extremidades (24\%) no tenían vasos que ingresen al pie. Run-off regular con solo
Tabla 1

CARACTERÍSTICAS CLÍNICAS DE LOS PACIENTES

\begin{tabular}{|c|c|c|c|c|}
\hline & $N$ & Media & Rango & $D S$ \\
\hline Edad & 54 & 70,6 & $(37-94)$ & 12,2 \\
\hline Sexo & \multicolumn{2}{|c|}{ Frecuencia } & \multicolumn{2}{|c|}{$\%$} \\
\hline Femenino & & 25 & \multicolumn{2}{|c|}{46} \\
\hline Masculino & & 29 & \multicolumn{2}{|c|}{54} \\
\hline Patologías Asociadas & & recuencia & \multicolumn{2}{|c|}{$\%$} \\
\hline Diabetes & & $40 / 54$ & \multicolumn{2}{|c|}{74} \\
\hline Hipertensión & & $38 / 54$ & \multicolumn{2}{|c|}{70} \\
\hline
\end{tabular}

un vaso en 28 (48\%); siendo en 12 de estos peronea, y bueno en 16 AGP $28 \%$ con salida en el pie por 2 o 3 vasos.

Las características clínicas de las extremidades sometidas a AGP se muestran en la Tabla 2.

El éxito técnico inicial se obtuvo en 49 procedimientos de un total de 58 (84\%).

Tabla 2

CARACTERÍSTICAS DE LAS EE.II.

\begin{tabular}{lrr}
\hline Características & $n$ & $\%$ \\
\hline Indicación AGP & & \\
Categoría Clínica 4 & 23 & $40 \%$ \\
Categoría Clínica 5 & 35 & $60 \%$ \\
TASC & & \\
Clasificación & & \\
Tipo A & 2 & $3,4 \%$ \\
Tipo B & 24 & $41,4 \%$ \\
Tipo C & 28 & $48,3 \%$ \\
Tipo D & 4 & $6,9 \%$ \\
Run-off & & \\
2 o 3 vasos & 16 & $28 \%$ \\
1 vaso & 28 & $48 \%$ \\
0 vasos & 14 & $24 \%$ \\
\hline
\end{tabular}

De las 9 AGP frustras dos extremidades fueron revascularizadas mediante by pass y 4 extremidades fueron amputadas. Los tres restantes corresponden a no candidatos a revascularización quirúrgica, y que rechazaron la amputación.

En este grupo existieron 2 complicaciones menores; perforación arterial evidenciada por extravasación del medio de contraste sin que se presentaran cambios en la condición clínica de las extremidades. No existió mortalidad, ni complicación mayor en estos 9 enfermos. 
De las AGP exitosas en 4 extremidades no hubo mejoría clínica ni hemodinámica inicial, a pesar de haber logrado anatómicamente un buen resultado, de estas dos tenían peronea como único vaso de salida y 2 no tenían vasos que entren al pie. El éxito clínico inicial fue de $91 \%$ (45/49) de las AGP exitosas y un $77,5 \%(45 / 58)$ del total de la muestra.

Hubo cuatro complicaciones mayores $6.8 \%$ (4/58)), un paciente sufre un Infarto Agudo de Miocardio por lo que fallece al $11^{\circ}$ día post operatorio, un paciente un AVE trombótico y dos trombosis del sitio Angioplastia que fueron solucionadas en forma abierta. La mortalidad del procedimiento fue un $1,7 \%(1 / 58)$.

En el grupo de AGP exitosas hubo 4 complicaciones menores $8 \%(4 / 49)$ ), dos hematomas, una perforación arterial auto controlada, y un paciente presenta pie de desecho, que se maneja con heparinización sistémica resolviéndose sin requerir cirugía.

El total de complicaciones fueron 4 mayores y 6 menores (7\% y $10 \%)$ en AGP exitosas y frustras.

Se repitió la Angioplastia en 4 extremidades, por presentarse a controles los pacientes con reaparición de los síntomas isquémicos y desaparición de pulsos antes presentes, confirmando la pérdida de permeabilidad con angiografía. No tenemos permeabilidad asistida ni secundaria ya que las extremidades fueron sometidas a re AGP cuando existía ya oclusión en el territorio estudiado; y con lesiones en distintos sectores de este.

Durante el seguimiento 5 pacientes fueron sometidos a by pass, un femorodistal y 4 femoropoplíteos, luego de oclusión de sus AGP.

En los 30 primeros días se realizaron $8 / 54$ (15\%) amputaciones mayores por ausencia de vasos dístales apropiados para cirugía y fracaso de la terapia endovascular. Al término del seguimiento hay 20 amputaciones mayores (34\%), siendo cuatro extremidades amputadas solo por compromiso local, uno de ellos con AGP ocluida y by pass permeable.

En el seguimiento durante el primer año posterior a la primera AGP fallecen 8 pacientes de los $54(17 \%)$, durante el segundo año 5, a los 3 años 2 y uno en el quinto año, final del seguimiento hay 16 pacientes fallecidos un $29,6 \%$ de mortalidad.

De los 54 enfermos iniciales a Abril 2006 permanecen 23 pacientes vivos y con la extremidad indemne, dos pacientes de este grupo fueron nuevamente sometidos a AGP después del cierre del estudio. Muertos y amputados 5 pacientes. Amputados y vivos 15 pacientes. Treinta y ocho pacientes vivos al cierre del seguimiento.

La permeabilidad primaria fue de 57,4 a 6 meses (ES 5,35), 43,3\% a 12 meses (ES 6,52), $32,7 \%$ a 24 meses (ES 7,44) y $16,9 \%$ a 36 meses (ES 6,31), 16,9\% a 48 meses (ES 10, 93), 16,9\% a 56 meses (ES 15,47). Tabla III

\section{DISCUSIÓN}

El papel de la Angioplastia en la isquemia crítica por patología ateromatosa del territorio fe-

Tabla 3

TABLA DE PERMEABILIDAD DE ANGIOPLASTIAS EXITOSAS

\begin{tabular}{|c|c|c|c|c|c|c|}
\hline $\begin{array}{c}\text { Intervalo en } \\
\text { meses }\end{array}$ & $\begin{array}{l}\text { Número de } \\
\text { angioplastias } \\
\text { exitosas al } \\
\text { comienzo del } \\
\text { intervalo }\end{array}$ & $\begin{array}{c}\text { Número de } \\
\text { fracasos } \\
\text { durante el } \\
\text { intervalo }\end{array}$ & $\begin{array}{c}\text { Fallecidos, } \\
\text { amputados o fin } \\
\text { de seguimiento } \\
\text { con angioplastia } \\
\text { permeable el } \\
\text { evento }\end{array}$ & $\begin{array}{c}\% \text { de fracaso } \\
\text { del intervalo }\end{array}$ & $\begin{array}{l}\% \text { de éxito } \\
\text { acumulado }\end{array}$ & Error standard \\
\hline$A$ & B & $\mathrm{C}$ & D & $E$ & $\mathrm{~F}$ & $\mathrm{G}$ \\
\hline $0-6$ & 49 & 20 & 4 & 42.5 & 57.4 & 5.35 \\
\hline $6-12$ & 25 & 6 & 1 & 24.4 & 43.3 & 6.52 \\
\hline $12-18$ & 18 & 3 & 2 & 17.6 & 35.7 & 6.75 \\
\hline $18-24$ & 13 & 1 & 2 & 8.3 & 32.7 & 7.44 \\
\hline $24-30$ & 10 & 2 & 2 & 22.2 & 25.4 & 6.95 \\
\hline $30-36$ & 6 & 2 & 0 & 33.3 & 16.9 & 6.31 \\
\hline $36-42$ & 4 & 0 & 2 & 0 & 16.9 & 7.73 \\
\hline $42-48$ & 2 & 0 & 1 & 0 & 16.9 & 10.93 \\
\hline $48-54$ & 1 & 0 & 1 & 0 & 16.9 & 15.47 \\
\hline
\end{tabular}


moropoplíteo, es aún controvertido, principalmente por la diversidad de los resultados informados, muy probablemente espejo de las diferencias en las características tanto de las extremidades como de los pacientes.

Asumiendo una baja permeabilidad, el poder ofrecer un procedimiento corto en tiempo operatorio, con estadía hospitalaria breve si el entorno lo permite, susceptible de realizar con anestesia local, y de baja morbimortalidad ${ }^{7,8}$ resulta altamente atractivo, principalmente si pensamos que es frecuente que el riesgo de morir en estos pacientes supere al riesgo de pérdida de la extremidad ${ }^{9-12}$ y que muchos de ellos no son candidatos a revascularización quirúrgica ya sea por condiciones generales o mal run off.

El éxito técnico inicial fue de un $84 \%$, dentro de lo esperado según el tipo de lesiones tratadas ${ }^{13}$ y el éxito clínico inicial también fue bajo lo que se asocia al número de extremidades con mal run off ${ }^{14}$.

Las complicaciones mayores la AGP del sector femoropoplíteo, varia entre 0 a $11 \% \%^{7,8,10}$, nuestra serie cae entre las cifras informadas y no existió amputación debida a complicación del procedimiento.

El alto porcentaje de amputaciones en el grupo puede deberse a que se incluyen extremidades sin run off, ausentes otras publicaciones con cifras mas bajas ${ }^{10}$.

Nuestra permeabilidad es similar a la de Löfberg $^{9}$, mejor que la de Parsons ${ }^{15}$ y es baja comparada con otras series ${ }^{10,16}$, lo que creemos es reflejo de las diferencias entre los grupos en estudio.

Debe recordarse que en este grupo de enfermos habitualmente el riesgo de morir supera al riesgo de amputación de la extremidad lo que se observa claramente en el BASIL ${ }^{12}$ y otras series ${ }^{10}$. En esta revisión, si excluimos aquellos pacientes que fueron amputados solo por compromiso local las cifras se igualan.

En conclusión es una terapia que no agrega riesgos importantes al paciente, manteniendo vigente la alternativa quirúrgica si esta fracasa, y que puede sacar de la isquemia incluso a pacientes no candidatos a cirugía convencional.

\section{REFERENCIAS}

1. Criqui $\mathrm{MH}$, Langer RD, Fronek $\mathrm{A}$, Feigelson HS, Klauber MR, McCann TJ. et al. Mortality over a period of 10 years in patients with peripheral arterial disease. N Engl J Med 1992; 326: 381-386.

2. Currie IC, Wakeley CJ, Cole SE, Wyatt MG, Scott DJ, Baid RN. et al. Femoro-popliteal angioplasty for severe limb ischaemia. Br J Surg 1994; 81: 191193.
3. Jansen $\mathrm{T}$, Manninen $\mathrm{H}$, Tulla $\mathrm{H}$, Matsi $\mathrm{P}$. The final outcome of primary infranguinal percutaneous transluminal angioplasty in 100 consecutive patients with chronic critical limb ischemia.- J Vasc Interv Radiol 2002; 13: 455-463.

4. Lepantalo M, Matzke S. Outcome of unreconstructed chronic critical leg ischaemia. Eur J Vasc Endovasc Surg 1996; 11: 153-157.

5. Rutherford RB, Baker JD, Ernst C. Recommended standards for reports dealing with lower extremity ischemia: revised version. J Vasc Surg 1997; 26: 517-538.

6. Dormandy JA, Rutherford RB. Managament of peripheral arterial disease (PAD). TASC Workin Group. TrasAtlantic Inter-Society Concensus (TASC). J Vasc Surg 2000; 31 (1Pt 2): S1-296.

7. Kudo T, Ahn SS. The effectiveness of percutaneous transluminal angioplasty for the treatment of critical limb ischemia: A 10-year experience. J Vasc Surg 2005; $31: 423$-435.

8. Hunink MG, Wong JB, Donaldson MC, Meyerovitz MF, de Vries J, Harrington DP. Revascularization for femoropopliteal disease. A decision and cost-effectiveness analysis. Jama 1995; 274: 165-171.

9. Lofberg AM, Karacagil S, Ljungman C, Westman B, BostromA, Hellberg A, et al. Percutaneous transluminal angioplasty of the femoropopliteal arteries in limbs with chronic critical lower limb ischemia. J Vasc Surg 2001: 34:114-121.

10. Martín-Álvarez A, González-Fueyo MJ, Malo E, Barbas-Galindo MJ, Ortega-Martín JM, Fernández-Morán $\mathrm{C}$ et al. Angioplastia transluminal percutánea del sector femoropoplíteo distal en la isquemia crítica. Angiologia 2004; 56: 154-162.

11. Nasr MK, McCarthy J RJ, Hardman, ChalmersA, Horrocks $M$. The increasing role of percutaneous transluminal angioplasty in the primary management of critical limb ischaemia. Eur J Vasc Endovasc Surg 2002; 23: 398-403.

12. Bypass versus angioplasty in severe ischaemia of the leg (BASIL):multicentre, randomized controlled trial. Lancet 2005; 366: 1925-1234.

13. Karch LA, Mattos MA, Henretta JP, McLafferty RB, Ramsey DE, Hodgson KJ. Clinical failure after percutaneous transluminal angioplasty of the superficial femoral and popliteal arteries. J Vasc Surg 2000; 31: 880-888.

14. Black JH, LaMuraglia GM, Kwolek CJ, Brewster DC, Watkins MT, Cambria RP. Contemporary results of angioplasty-based infrainguinal percutaneous interventions. J Vasc Surg 2005; 42: 932-937.

15. Parsons RE, Suggs WD, Lee JJ, Sanchez LA, Lyon RT, Veith FJ. Percutaneous Transluminal Angioplasty for the treatment: of limb threatening ischemia: do the results justify an attempt before bypass grafting? J Vasc Surg 1998; 28: 1066-1071.

16. Conrad MF, Cambria R, Stone D, Brewster D, Kwolek $\mathrm{C}$, Watkins $\mathrm{M}$. et al. Intermediate results of percutaneus endovascular therapy of femoropopliteal occlusive disease: A contemporary series J Vasc Surg 2006; 44: 762-769. 